\title{
SEMIDISCRETIZATION FOR A NONLOCAL PARABOLIC PROBLEM
}

\author{
ABDERRAHMANE EL HACHIMI AND MOULAY RCHID SIDI AMMI
}

Received 22 February 2004 and in revised form 16 February 2005

A time discretization technique by Euler forward scheme is proposed to deal with a nonlocal parabolic problem. Existence and uniqueness of the approximate solution are proved.

\section{Introduction}

In this work, we study the time discretization by Euler forward scheme of the nonlocal initial boundary value problem

$$
\begin{gathered}
\left.\frac{\partial u}{\partial t}-\Delta u=\lambda \frac{f(u)}{\left(\int_{\Omega} f(u) d x\right)^{2}} \quad \text { in } \Omega \times\right] 0 ; T[ \\
u=0 \quad \text { on } \partial \Omega \times] 0 ; T[ \\
u(0)=u_{0} \quad \text { in } \Omega
\end{gathered}
$$

with $\Omega \subset \mathbb{R}^{d}(d \geq 1)$ a bounded regular domain and $\lambda$ a positive parameter. The hypotheses we will assume on $f$ are the same as in [6]. We recall first that (1.1) arises by reducing the following system of two equations modeling the thermistor problem:

$$
\begin{gathered}
u_{t}=\nabla \cdot(k(u) \nabla u)+\sigma(u)|\nabla \varphi|^{2}, \\
\nabla(\sigma(u) \nabla \varphi)=0,
\end{gathered}
$$

where $u$ represents the temperature generated by the electric current flowing through a conductor, $\varphi$ the electric potential, $\sigma(u)$ and $k(u)$ are, respectively, the electric and thermal conductivities. For more description, we refer to $[5,6,7,8,11]$ among others.

We recall also that the Euler forward method was used by several authors to treat semidiscretization of nonlinear parabolic problems, see $[3,4]$. Concerning problem (1.1), results of existence and uniqueness of solutions are known under particular forms of $f$, we refer to [2] and the references therein. On the other hand, little is known about 


\section{Semidiscretization for a nonlocal parabolic problem}

the solutions to the discrete problem

$$
\begin{gathered}
U^{n}-\tau \Delta U^{n}=U^{n-1}+\lambda \tau \frac{f\left(U^{n}\right)}{\left(\int_{\Omega} f\left(U^{n}\right) d x\right)^{2}} \quad \text { in } \Omega, \\
U^{n}=0 \quad \text { on } \partial \Omega, \\
U^{0}=u_{0} \quad \text { in } \Omega .
\end{gathered}
$$

Whereas, semidiscretization has been involved for the equations of the thermistor problem in $[1,9]$. Our aim here is to continue the study of problem (1.1) initiated in [6], where an a priori $L^{\infty}$-estimate is derived. In addition to habitual existence and uniqueness questions concerning the solutions of (1.3), we will prove some results of stability and proceed to error estimates analysis. In [1], the authors derived an $L^{2}$ and $H^{1}$-norm error by requiring more regularity on the solution $u$, for instance $u, u_{t}$ in $H^{2}(\Omega) \cap W^{1, \infty}(\Omega)$. Unfortunately, such smoothness is not always possible since the function $f$ is nonlinear.

\section{The semidiscrete problem}

2.1. Existence and uniqueness. We consider the Euler scheme (1.3), with $N \tau=T, T>0$ fixed, and $1 \leq n \leq N$, under the following hypotheses.

(H1) $f: \mathbb{R} \rightarrow \mathbb{R}$ is a locally Lipschitzian function.

(H2) There exist positive constants $\sigma, c_{1}, c_{2}$, and $\alpha$ such that $\alpha<4 /(d-2)$ and for all $\xi \in \mathbb{R}$

$$
\sigma \leq f(\xi) \leq c_{1}|\xi|^{\alpha+1}+c_{2}
$$

In the sequel, we will denote the norms in the spaces $L^{\infty}(\Omega), L^{k}(\Omega)$ by $|\cdot|_{L^{\infty}(\Omega)}$ and $|\cdot|_{k}$, respectively, $(\cdot, \cdot)$ will denote the associated inner product in $L^{2}(\Omega)$ or the duality product between $H_{0}^{1}(\Omega)$ and its dual $H^{-1}(\Omega)$.

Theorem 2.1. Let (H1)-(H2) be satisfied. Then, for each $n$, there exists a unique solution $U^{n}$ of $(1.3)$ in $H_{0}^{1}(\Omega) \cap L^{\infty}(\Omega)$ provided that $\tau$ is small enough.

Proof. For simplicity, we write $U=U^{n}, h(x)=U^{n-1}$. Then (1.3) becomes

$$
\begin{gathered}
U-\tau \Delta U=h(x)+\lambda \frac{f(U)}{\left(\int_{\Omega} f(U) d x\right)^{2}} \quad \text { in } \Omega, \\
U=0 \quad \text { on } \partial \Omega .
\end{gathered}
$$

Existence. Define the map $S(\mu, \cdot)$ by $U=S(\mu, v), \mu \in[0,1]$ if and only if

$$
\begin{gathered}
U-\tau \triangle U=\mu g(x, v) \quad \text { in } \Omega, \\
U=0 \quad \text { on } \partial \Omega, \\
U^{0}=\mu u_{0},
\end{gathered}
$$


where $g(x, v)=h(x)+\lambda\left(f(v) /\left(\int_{\Omega} f(v) d x\right)^{2}\right)$. For a fixed $v \in H_{0}^{1}(\Omega)$, (2.3) has a unique solution $U \in H_{0}^{1}(\Omega)$. Then, for each $\mu \in[0,1]$, the operator $S(\mu, \cdot)$ is well defined. Moreover, $S(\mu, \cdot)$ is compact from $H_{0}^{1}(\Omega)$ into it self. Indeed, using $(\mathrm{H} 2)$, we have the estimate

$$
|U|_{2}^{2}+\tau|\nabla U|_{2}^{2} \leq c_{3}
$$

We can easily see that $\mu \rightarrow S(\mu, v)$ is continuous and that $S(0, v)=U$, for any $v$, if and only if $U=0$. From Leray-Schauder fixed point theorem, there exists therefore a fixed point $U$ of $S(\mu, \cdot)$.

Now, we derive an a priori estimate.

Lemma 2.2. If $u_{0} \in L^{\infty}(\Omega)$, then for all $n \in\{1, \ldots, N\}, U^{n} \in L^{\infty}(\Omega)$.

Proof. The proof is similar to the one used by De Thélin in [10] concerning a very different problem and we will give here only a sketch. Suppose that $d \geq 2$ and define

$$
\delta= \begin{cases}\frac{2 d}{d-2} & \text { if } 2<d, \\ 2(\alpha+2) & \text { if } d=2 .\end{cases}
$$

For each $k \in \mathbb{N} *$, we consider the number

$$
\begin{gathered}
q_{k}=\left\{\left(\frac{\delta}{2}\right)^{k-1}(\delta-\gamma)-(2-\gamma)\right\} \frac{\delta}{\delta-2}, \quad k \geq 2, \\
q_{1}=\delta
\end{gathered}
$$

we have

$$
q_{k+1}=\left(q_{k}+2-\gamma\right) \frac{\delta}{2} \quad \text { with } \gamma=\alpha+2, \forall k \in \mathbb{N}^{*}
$$

Lemma 2.3. For all $k \in \mathbb{N}^{*}, U^{n} \in L^{q_{k}}(\Omega)$, and moreover

$$
\left|U^{n}\right|_{\infty}=\varlimsup \lim \left|U^{n}\right|_{q k}<+\infty
$$

Proof. We prove by recurrence that $U \in L^{q_{k}}$. The property is true for $k=1$, since $H_{0}^{1}(\Omega) \subset$ $L^{\delta}(\Omega)$. We show now that $U \in L^{q_{k+1}}$. Let $m \in \mathbb{N}, 1 \leq m \leq k$. Multiplying (2.2) by $|U|^{q_{m}-\gamma} U$, using $(\mathrm{H} 2)$, and Young's inequality, we get

$$
\left(q_{m}-\gamma+1\right) \int_{\Omega}|\nabla U|^{2}|U|^{q_{m}-\gamma} d x \leq c_{4}|U|_{q_{m}}^{q_{m}}+c_{5}
$$

On the other hand, we have

$$
|U|_{q_{m+1}}^{q_{m}+2-\gamma} \leq c_{6}\left(1+\frac{q_{m}-\gamma}{2}\right)^{2} \int_{\Omega}|\nabla U|^{2}|U|^{q_{m}-\gamma} d x .
$$


1658 Semidiscretization for a nonlocal parabolic problem

Therefore, we obtain

$$
|U|_{q_{m+1}}^{q_{m}+2-\gamma} \leq\left(c_{7}+c_{8}|U|_{q_{m}}^{q_{m}}\right)\left(q_{m}+2-\gamma\right)
$$

Thus,

$$
\left(|U|_{q_{k+1}}^{q_{k+1}}\right)^{2 / \delta} \leq\left(c_{7}+c_{8}|U|_{q_{k}}^{q_{k}}\right)\left(q_{k}+2-\gamma\right)
$$

The rest of the proof follows the same lines as in [10, pages 383-384].

Uniqueness. Consider $U$ and $V$ two different solutions of (2.2) and define $w=U-V$. Then, we have

$$
\begin{aligned}
w-\tau \Delta w= & \frac{\lambda \tau}{\left(\int_{\Omega} f(U) d x\right)^{2}}(f(U)-f(V)) \\
& +\lambda \tau \frac{\left(\int_{\Omega} f(U)-f(V) d x\right)\left(\int_{\Omega} f(V)+f(U) d x\right)}{\left(\int_{\Omega} f(U) d x\right)^{2}\left(\int_{\Omega} f(V) d x\right)^{2}} f(V) .
\end{aligned}
$$

Multiplying (2.13) by $w$, integrating on $\Omega$, and using the $L^{\infty}$-estimate obtained in Lemma 2.2 , we get

$$
|w|_{2}^{2}+\tau|\nabla w|_{2}^{2} \leq c_{9} \tau|w|_{2}^{2}
$$

Therefore, $w=0$ if $\tau \leq 1 / c_{9}$.

We address now the question of stability.

\section{Stability}

Theorem 3.1. Assume (H1)-(H2) hold. Then, there exists $c\left(T, u_{0}\right)>0$ depending on data but not on $N$ such that for any $n \in\{1, \ldots, N\}$,

(a) $\left|U^{n}\right|_{L^{\infty}(\Omega)} \leq c\left(T, u_{0}\right)$;

(b) $\left|U^{n}\right|_{2}^{2}+\tau \sum_{k=1}^{n}\left|\nabla U^{k}\right|_{2}^{2} \leq c\left(T, u_{0}\right)$;

(c) $\sum_{k=1}^{n}\left|U^{k}-U^{k-1}\right|_{2}^{2} \leq c\left(T, u_{0}\right)$.

Proof. (i) Multiplying (1.3) by $\left|U^{k}\right|^{m} U^{k}$ for some integer $m \geq 1$, using Lemma 2.2, and Hölder's inequality, we obtain after simplification

$$
\left|U^{k}\right|_{m+2} \leq\left|U^{k-1}\right|_{m+2}+c_{10} \tau
$$

By induction and taking the limit in the resulting inequality as $m \rightarrow+\infty$, we get

$$
\left|U^{k}\right|_{L^{\infty}(\Omega)} \leq c\left(T, u_{0}\right) .
$$

(ii) Multiplying the first equation of (1.3) by $U^{k}$ and using the hypotheses on $f$, one easily has

$$
\left(U^{k}-U^{k-1}, U^{k}\right)+\tau\left|\nabla U^{k}\right|_{2}^{2} \leq c_{11} \tau\left|U^{k}\right|_{1}
$$


Using the elementary identity $2 a(a-b)=a^{2}-b^{2}+(a-b)^{2}$ and summing from $k=1$ to $n$, we obtain

$$
\left|U^{n}\right|_{2}^{2}+\sum_{k=1}^{n}\left|U^{k}-U^{k-1}\right|_{2}^{2}+\tau \sum_{k=1}^{n}\left|\nabla U^{k}\right|_{2}^{2} \leq\left|u_{0}\right|_{2}^{2}+\tau c_{12} \sum_{k=1}^{n}\left|U^{k}\right|_{1} .
$$

Then, the inequalities (b)-(c) hold by using the uniform bound of $U^{n}$ in $L^{\infty}$ which is established in part (a).

\section{Error estimates for solutions}

We will adopt the following notations concerning the time discretization for problem (1.1). We denote the time step $\tau=T / N, t^{n}=n \tau$, and $I_{n}=\left(t^{n}, t^{n-1}\right)$ for $n=1, \ldots, N$. If $z$ is a continuous function (resp., summable), defined in $(0, T)$ with values in $H^{-1}(\Omega)$ or $L^{2}(\Omega)$ or $H_{0}^{1}(\Omega)$, we define $z^{n}=z\left(t^{n}, \cdot\right), \bar{z}^{n}=(1 / \tau) \int_{I_{n}} z(t, \cdot) d t, \bar{z}^{0}=z^{0}=z(0, \cdot)$; the error $e_{n}=u(t)-U^{n}$ for all $t \in I_{n}$ and the local errors $e_{u}^{n}$ and $e^{n}$ defined by $e_{u}^{n}=\bar{u}^{n}(t)-U^{n}$, $e^{n}=u^{n}-U^{n}$.

We have the following theorem.

Theorem 4.1. Let (H1)-(H2) hold. Then, the following error bounds are satisfied:

(1) $\left\|e_{n}\right\|_{L^{\infty}\left(0, T, H^{-1}(\Omega)\right)}^{2}+\int_{0}^{T}\left|e_{n}\right|^{2} d t \leq c_{13} \tau$,

(2) $\left\|e^{m}\right\|_{H^{-1}(\Omega)} \leq c_{14} \tau^{1 / 2}$,

(3) $\left|\nabla \int_{0}^{T} e_{n}(t) d t\right|_{2} \leq c_{15} \tau^{1 / 4}$.

Proof. For the proof, we consider the following variational formulation of discrete problem (1.3):

$$
\left(U^{n}-U^{n-1}, \varphi\right)+\tau\left(\nabla U^{n}, \nabla \varphi\right)=\frac{\lambda \tau}{\left(\int_{\Omega} f\left(U^{n}\right) d x\right)^{2}}\left(f\left(U^{n}\right), \varphi\right), \quad \forall \varphi \in H_{0}^{1}(\Omega) .
$$

Integrating the continuous problem (1.1) over $I_{n}$, we get

$$
\left(u^{n}-u^{n-1}, \varphi\right)+\tau\left(\nabla \bar{u}^{n}, \nabla \varphi\right)=\lambda \tau \frac{\left(\overline{f\left(u^{n}\right)}, \varphi\right)}{\left(\int_{\Omega} f\left(u^{n}\right) d x\right)^{2}}, \quad \forall \varphi \in H_{0}^{1}(\Omega)
$$

Substracting (4.2) from (4.1) and adding from $n=1$ to $m$ with $m \leq N$, we obtain

$$
\begin{aligned}
& \sum_{n=1}^{m}\left(e^{n}-e^{n-1}, \varphi\right)+\tau \sum_{n=1}^{m}\left(\nabla e_{u}^{n}, \nabla \varphi\right) \\
& \quad \leq c_{16} \tau\left|\sum_{n=1}^{m}\left(\overline{f(u}^{n}-f\left(U^{n}\right), \varphi\right)\right|+c_{17} \tau\left|\sum_{n=1}^{m}\left(f\left(U^{n}\right), \varphi\right)\right| .
\end{aligned}
$$


1660 Semidiscretization for a nonlocal parabolic problem

Let $(-\triangle)^{-1}$ be the green operator satisfying

$$
\left(\nabla(-\triangle)^{-1} \psi, \nabla \varphi\right)=(\psi, \varphi)_{H^{-1}(\Omega), H_{0}^{1}(\Omega)}
$$

for all $\psi \in H^{-1}(\Omega), \varphi \in H_{0}^{1}(\Omega)$. Choosing $\varphi=(-\triangle)^{-1}\left(e^{n}\right)$ as test function, we then obtain

$$
I_{1}+I_{2} \leq I_{3}+I_{4}
$$

where

$$
\begin{aligned}
& I_{1}=\sum_{n=1}^{m}\left(e^{n}-e^{n-1},(-\triangle)^{-1}\left(e^{n}\right)\right), \\
& I_{2}=\tau \sum_{n=1}^{m}\left(e_{u}^{n}, e^{n}\right), \\
& I_{3} \leq c_{16} \tau\left|\sum_{n=1}^{m}\left(\overline{f(u}^{n}-f\left(U^{n}\right),(-\triangle)^{-1}\left(e^{n}\right)\right)\right|, \\
& I_{4}=c_{17} \tau\left|\sum_{n=1}^{m}\left(f\left(U^{n}\right),(-\triangle)^{-1}\left(e^{n}\right)\right)\right| .
\end{aligned}
$$

With the aid of the elementary identity $2 a(a-b)=a^{2}-b^{2}+(a-b)^{2}$ and the property of $(-\triangle)^{-1}, I_{1}$ reduces after straightforward calculations to

$$
I_{1}=\frac{1}{2}\left\|e^{m}\right\|_{H^{-1}(\Omega)}^{2}+\frac{1}{2} \sum_{n=1}^{m}\left\|e^{n}-e^{n-1}\right\|_{H^{-1}(\Omega)}^{2} .
$$

On the other hand,

$$
\begin{aligned}
I_{2} & =\tau \sum_{n=1}^{m}\left(e_{u}^{n}, e^{n}\right) \\
& =\sum_{n=1}^{m} \int_{I_{n}}\left(u(t)-U^{n}, u(t)-U^{n}\right) d t+\sum_{n=1}^{m} \int_{I_{n}}\left(u(t)-U^{n}, u^{n}-u(t)\right) d t \\
& =I_{21}+I_{22},
\end{aligned}
$$

where

$$
\begin{aligned}
I_{21} & =\sum_{n=1}^{m} \int_{I_{n}}\left(u(t)-U^{n}, u(t)-U^{n}\right) d t=\sum_{n=1}^{m} \int_{I_{n}}\left|e_{n}\right|_{2}^{2} d t \\
I_{22} & =\sum_{n=1}^{m} \int_{I_{n}}\left(u(t), u^{n}-u(t)\right) d t-\sum_{n=1}^{m} \int_{I_{n}}\left(U^{n}, u^{n}-u(t)\right) d t \\
& =I_{22}^{1}+I_{22}^{2} .
\end{aligned}
$$


We now estimate $I_{22}^{1}$. Using the boundedness of $\partial u / \partial s$ (see [6]), we have

$$
\begin{aligned}
\left|I_{22}^{1}\right| & =\left|\sum_{n=1}^{m} \int_{I_{n}}\left(u(t), \int_{t}^{t^{n}} \frac{\partial u}{\partial s} d s\right) d t\right| \\
& \leq \sum_{n=1}^{m} \int_{I_{n}}\left(\int_{t}^{t^{n}}\left\|\frac{\partial u}{\partial s}\right\|_{H^{-1}(\Omega)} d s\right)\|u(t)\|_{H_{0}^{1}(\Omega)} d t \\
& \leq \tau \mid\left\|\frac{\partial u}{\partial s}\right\|_{L^{2}\left(0, t^{m}, H^{-1}(\Omega)\right)}\|u\|_{L^{2}\left(0, t^{m}, H_{0}^{1}(\Omega)\right)} \\
& \leq c_{18} \tau .
\end{aligned}
$$

In the same manner, we have

$$
\begin{aligned}
\left|I_{22}^{2}\right| & \leq \tau \mid\left\|\frac{\partial u}{\partial s}\right\| \|_{L^{2}\left(0, t^{m}, H^{-1}(\Omega)\right)}\left(\tau \sum_{n=1}^{m}\left\|U^{n}\right\|_{H_{0}^{1}(\Omega)}^{2}\right)^{1 / 2} \\
& \leq c_{18} \tau .
\end{aligned}
$$

Next, we estimate the first term on the right-hand side of (4.5) by using Hölder's and Young's inequalities and (H1),

$$
\begin{aligned}
\left|I_{3}\right| & \leq\left|\sum_{n=1}^{m}\left(\int_{I_{n}}\left[f(u)-f\left(U^{n}\right)\right] d t,(-\triangle)^{-1}\left(e^{n}\right)\right)\right| \\
& \leq c_{20} \tau^{1 / 2} \sum_{n=1}^{m}\left(\int_{I_{n}}\left|f(u)-f\left(U^{n}\right)\right|_{2}^{2} d t\right)^{1 / 2}\left\|e^{n}\right\|_{H^{-1}(\Omega)} \\
& \leq \eta \sum_{n=1}^{m}\left(\int_{I_{n}}\left|f(u)-f\left(U^{n}\right)\right|_{2}^{2} d t\right)+\frac{c_{21}}{\eta} \tau \sum_{n=1}^{m}\left\|e^{n}\right\|_{H^{-1}(\Omega)}^{2} \\
& \leq c_{22} \eta \sum_{n=1}^{m}\left(\int_{I_{n}}\left|e_{n}\right|_{2}^{2} d t\right)+\frac{c_{21}}{\eta} \tau \sum_{n=1}^{m}\left\|e^{n}\right\|_{H^{-1}(\Omega)}^{2} .
\end{aligned}
$$

Moreover, we have

$$
\left|I_{4}\right| \leq c_{23} \tau+c_{24} \tau \sum_{n=1}^{m}\left\|e^{n}\right\|_{H^{-1}(\Omega)}^{2} .
$$

Choosing suitable $\eta$, we conclude that

$$
\begin{aligned}
& \left\|e^{m}\right\|_{H^{-1}(\Omega)}^{2}+\sum_{n=1}^{m}\left\|e^{n}-e^{n-1}\right\|_{H^{-1}(\Omega)}^{2}+\sum_{n=1}^{m} \int_{I_{n}}\left|e_{n}\right|_{2}^{2} d t \\
& \leq c_{25} \tau+c_{26} \tau \sum_{n=1}^{m}\left\|e^{n}\right\|_{H^{-1}(\Omega)}^{2} .
\end{aligned}
$$

On the other hand, setting $y^{m}=\sum_{n=1}^{m}\left\|e^{n}\right\|_{H^{-1}(\Omega)}^{2}$, then from (4.14), we get

$$
y^{m}-y^{m-1} \leq c_{25} \tau+c_{26} \tau y^{m} .
$$


1662 Semidiscretization for a nonlocal parabolic problem

By applying the discrete Gronwall inequality, we deduce that $y^{m} \leq c(T)$. Therefore, we have

$$
\left\|e^{m}\right\|_{H^{-1}(\Omega)} \leq c_{27} \tau^{1 / 2}
$$

On the other hand, we have

$$
\sup _{t \in\left(0, t_{m}\right)}\left\|e_{n}(t)\right\|_{H^{-1}(\Omega)}-c_{27} \tau^{1 / 2} \leq \max _{1 \leq n \leq m}\left\|e_{n}\left(t^{n}\right)\right\|_{H^{-1}(\Omega)}=\max _{1 \leq n \leq m}\left\|e^{n}\right\|_{H^{-1}(\Omega)} .
$$

Thus, we get

$$
\left\|e_{n}\right\|_{L^{\infty}\left(0, T, H^{-1}(\Omega)\right)}-c_{27} \tau^{1 / 2} \leq \max _{1 \leq n \leq m}\left\|e^{n}\right\|_{H^{-1}(\Omega)}
$$

From the last inequality, we obtain

$$
\begin{gathered}
\left\|e_{n}\right\|_{L^{\infty}\left(0, T, H^{-1}(\Omega)\right)}^{2}+\int_{0}^{T}\left|e_{n}\right|_{2}^{2} d t \leq c_{29} \tau \\
\sum_{n=1}^{m}\left\|e^{n}-e^{n-1}\right\|_{H^{-1}(\Omega)}^{2} \leq c_{29} \tau
\end{gathered}
$$

Choosing $\varphi=\tau \sum_{n=1}^{m}\left(\bar{u}^{n}-U^{n}\right)$ in (4.3), we get

$$
\begin{aligned}
\tau \int_{\Omega}( & \left.u^{m}-U^{m}\right)\left(\sum_{n=1}^{m}\left(\bar{u}^{n}-U^{n}\right) d x\right)+\tau^{2}\left|\sum_{n=1}^{m} \nabla\left(\bar{u}^{n}-U^{n}\right)\right|_{2}^{2} \\
\leq & c_{30} \tau^{2}\left|\int_{\Omega} \sum_{n=1}^{m}\left(\overline{f(u)}^{n}-f\left(U^{n}\right)\right)\left(\sum_{n=1}^{m}\left(\bar{u}^{n}-U^{n}\right)\right) d x\right| \\
& +c_{31} \tau^{2}\left|\sum_{n=1}^{m}\left(f\left(U^{n}\right), \sum_{n=1}^{m}\left(\bar{u}^{n}-U^{n}\right)\right)\right|
\end{aligned}
$$

Thus,

$$
\begin{aligned}
\tau^{2}\left|\sum_{n=1}^{m} \nabla\left(\bar{u}^{n}-U^{n}\right)\right|_{2}^{2}= & \left|\nabla \int_{0}^{t^{m}} e_{n} d t\right|_{2}^{2} \leq \tau\left|\int_{\Omega}\left(u^{m}-U^{m}\right)\left(\sum_{n=1}^{m}\left(\bar{u}^{n}-U^{n}\right) d x\right)\right| \\
& +c_{30} \tau^{2}\left|\int_{\Omega} \sum_{n=1}^{m}\left(\overline{f(u)}^{n}-f\left(U^{n}\right)\right)\left(\sum_{n=1}^{m}\left(\bar{u}^{n}-U^{n}\right)\right) d x\right| \\
& +c_{31} \tau^{2}\left|\sum_{n=1}^{m}\left(f\left(U^{n}\right), \sum_{n=1}^{m}\left(\bar{u}^{n}-U^{n}\right)\right)\right| \\
\leq & I+I I+I I I .
\end{aligned}
$$


Clearly,

$$
\begin{aligned}
I & \leq\left\|e^{m}\right\|_{H^{-1}(\Omega)}\left(\sum_{n=1}^{m} \int_{I_{n}}\|u(t)\|_{H_{0}^{1}(\Omega)} d t+\tau \sum_{n=1}^{m}\left\|U^{n}\right\|_{H_{0}^{1}(\Omega)}\right) \\
& \leq c_{32}\left\|e^{m}\right\|_{H^{-1}(\Omega)} \\
& \leq c_{33} \tau^{1 / 2} .
\end{aligned}
$$

We also get

$$
\begin{aligned}
I I & \leq\left(\int_{\Omega}\left(\sum_{n=1}^{m} \int_{I_{n}}\left(f(u)-f\left(U^{n}\right)\right) d t\right)^{2} d x\right)^{1 / 2} \times\left(\int_{\Omega}\left(\sum_{n=1}^{m} \int_{I_{n}}\left(u(t)-U^{n}\right) d t\right)^{2} d x\right)^{1 / 2} \\
& \leq T^{2}\left(\sum_{n=1}^{m} \int_{I_{n}}\left|f(u)-f\left(U^{n}\right)\right|_{2}^{2} d t\right)^{1 / 2} \times\left(\sum_{n=1}^{m} \int_{I_{n}}\left|u(t)-U^{n}\right|_{2}^{2} d t\right)^{1 / 2} \\
& \leq T^{2}\left(\sum_{n=1}^{m} \int_{I_{n}}\left|f(u)-f\left(U^{n}\right)\right|_{2}^{2} d t\right)^{1 / 2} \times\left(2\|u\|_{L^{2}\left(0, T, H_{0}^{1}(\Omega)\right)}^{2}+2 \tau \sum_{n=1}^{m}\left|U^{n}\right|_{2}^{2}\right)^{1 / 2} \\
& \leq c_{34} \tau^{1 / 2} .
\end{aligned}
$$

The last inequality follows by using simultaneously the $L^{\infty}$-estimate of $u(t)$ (see [6]), $U^{n}$, and the error bound given in (a). Arguing exactly as in the previous estimate, we get

$$
I I I \leq T^{2}\left(\sum_{n=1}^{m} \int_{I_{n}}\left|f\left(U^{n}\right)\right|_{2}^{2} d t\right)^{1 / 2} \times\left(2\|u\|_{L^{2}\left(0, T, H_{0}^{1}(\Omega)\right)}^{2}+2 \tau \sum_{n=1}^{m}\left|U^{n}\right|_{2}^{2}\right)^{1 / 2} .
$$

Using again the hypothesis (H1) and the estimates above, we obtain

$$
I I I \leq c_{35} \tau^{1 / 2}
$$

Finally collecting these results, it follows that

$$
\left|\nabla \int_{0}^{T} e_{n} d t\right|_{2}^{2} \leq c_{36} \tau^{1 / 2}
$$

This completes the proof.

Corollary 4.2. Under hypotheses (H1)-(H2), problem (1.3) generates a continuous semigroup $S_{\tau}$ defined by $S_{\tau} U^{n-1}=U^{n}$.

\section{References}

[1] W. Allegretto, Y. Lin, and A. Zhou, A box scheme for coupled systems resulting from microsensor thermistor problems, Dynam. Contin. Discrete Impuls. Systems 5 (1999), no. 1-4, 209-223.

[2] J. W. Bebernes and A. A. Lacey, Global existence and finite-time blow-up for a class of nonlocal parabolic problems, Adv. Differential Equations 2 (1997), no. 6, 927-953. 


\section{Semidiscretization for a nonlocal parabolic problem}

[3] F. Benzekri and A. El Hachimi, Doubly nonlinear parabolic equations related to the p-Laplacian operator: semi-discretization, Electron. J. Differential Equations 2003 (2003), no. 113, 1-14.

[4] A. Eden, B. Michaux, and J.-M. Rakotoson, Semi-discretized nonlinear evolution equations as discrete dynamical systems and error analysis, Indiana Univ. Math. J. 39 (1990), no. 3, 737783.

[5] A. El Hachimi and M. R. Sidi Ammi, Existence of weak solutions for the thermistor problem with degeneracy, Proceedings of the 2002 Fez Conference on Partial Differential Equations, Electron. J. Differ. Equ. Conf., vol. 9, Southwest Texas State University, Texas, 2002, pp. $127-137$.

[6] _ Existence of global solution for a nonlocal parabolic problem, Electron. J. Qual. Theory Differ. Equ. 2005 (2005), no. 1, 1-9.

[7] A. A. Lacey, Thermal runaway in a non-local problem modelling Ohmic heating. I. Model derivation and some special cases, European J. Appl. Math. 6 (1995), no. 2, 127-144.

[8] - Thermal runaway in a non-local problem modelling Ohmic heating. II. General proof of blow-up and asymptotics of runaway, European J. Appl. Math. 6 (1995), no. 3, 201-224.

[9] P. Shi, M. Shillor, and X. Xu, Existence of a solution to the Stefan problem with Joule's heating, J. Differential Equations 105 (1993), no. 2, 239-263.

[10] F. de Thélin, Résultats d'existence et de non-existence pour la solution positive et bornée d'une e.d.p. elliptique non linéaire [Existence and nonexistence results for a positive bounded solution of a nonlinear elliptic partial differential equation], Ann. Fac. Sci. Toulouse Math. (5) 8 (1986/1987), no. 3, 375-389 (French).

[11] D. E. Tzanetis, Blow-up of radially symmetric solutions of a non-local problem modelling Ohmic heating, Electron. J. Differential Equations 2002 (2002), no. 11, 1-26.

Abderrahmane El Hachimi: UFR Mathématiques Appliquées et Industrielles, Département de Mathématiques et Informatique, Faculté des Sciences, Université Chouaib Doukkali, B.P. 20, El Jadida, Morocco

E-mail address: elhachimi@ucd.ac.ma

Moulay Rchid Sidi Ammi: UFR Mathématiques Appliquées et Industrielles, Département de Mathématiques et Informatique, Faculté des Sciences, Université Chouaib Doukkali, B.P. 20, El Jadida, Morocco

E-mail address: rachidsidiammi@yahoo.fr 


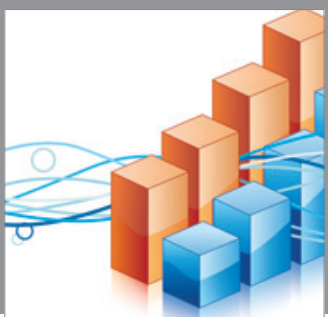

Advances in

Operations Research

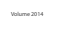



\section{The Scientific} World Journal
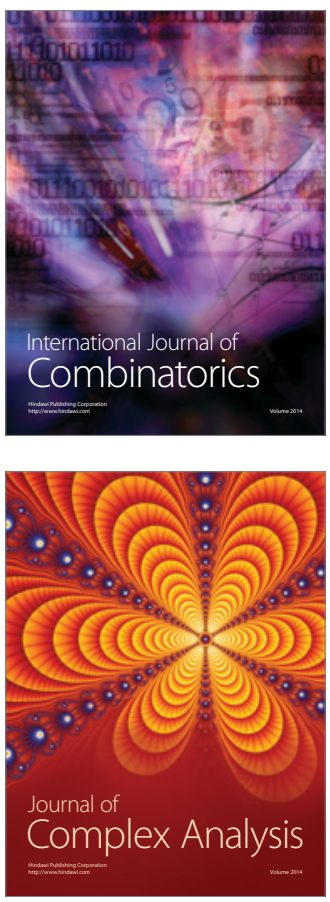

International Journal of

Mathematics and

Mathematical

Sciences


Journal of

Applied Mathematics
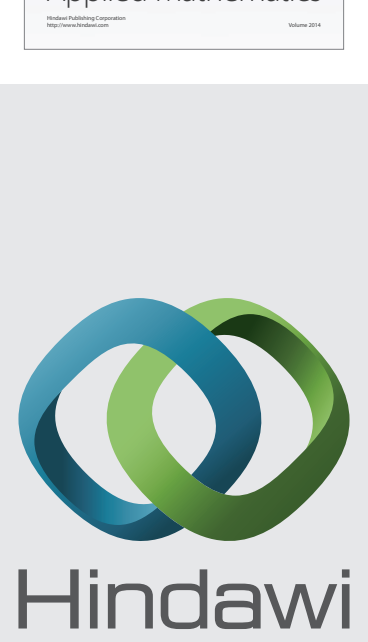

Submit your manuscripts at http://www.hindawi.com
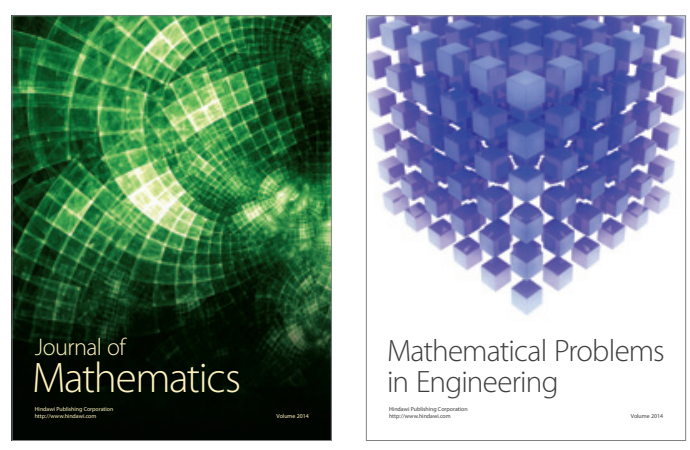

Mathematical Problems in Engineering
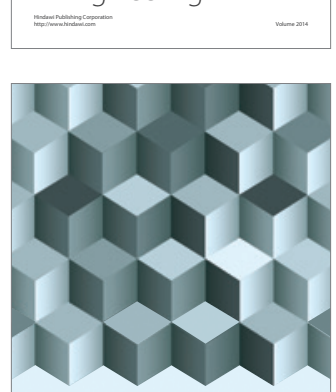

Journal of

Function Spaces
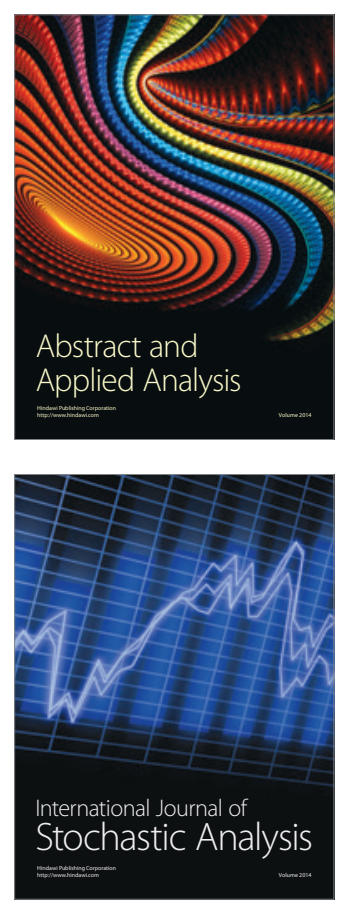



ournal of

Probability and Statistics

Promensencen
